\title{
Diels-Alder reaction between indoles and cyclohexadienes photocatalyzed by a (thia)pyrylium salt
}

\author{
María González-Béjar, ${ }^{a}$ Salah-Eddine Stiriba, ${ }^{a}$ Miguel A. Miranda, ${ }^{b, *}$ \\ and Julia Pérez-Prieto ${ }^{\text {a,* }}$ \\ ${ }^{a}$ Departamento de Química Orgánica/Instituto de Ciencia Molecular, Universidad de Valencia, \\ Polígono La Coma, 46980, Paterna, Valencia, Spain \\ ${ }^{b}$ Departamento de Química/Instituto de Tecnología Química UPV-CSIC; Universidad \\ Politécnica de Valencia, Camino de Vera s/n, 46022, Valencia, Spain \\ E-mail: julia.perez@uv.es
}

Dedicated to Prof. Joan Bosch on the occasion of his $60^{\text {th }}$ anniversary

\begin{abstract}
Thiapyrylium salt $\mathbf{1}$ is an efficient photocatalyst in the Diels-Alder reaction between indoles (InHs) and 1,3-cyclohexadienes (CHDs). For instance, irradiation of deaerated dichloromethane solutions containing $1(1 \mathrm{mM})$, indole $(20 \mathrm{mM})$ and cyclohexadiene $(40 \mathrm{mM})$, in the presence of an acylating agent, led to the Diels-Alder cycloadduct in $62 \%$ yield (endo:exo ratio of 1.8:1). Taking into account the very high intersystem crossing yield $\left(\Phi_{\text {isc }}=0.97\right)$ of thiapyrylium salt $\mathbf{1}$, involvement of its triplet excited state in this Diels-Alder reaction has been studied using steadystate and time-resolved experiments; the results are compared with those previously obtained for pyrylium salts, which exhibit a considerable fluorescence quantum yield and are assumed to operate via electron transfer from the InH to their singlet excited state.
\end{abstract}

Keywords: Diels-Alder reaction, photocatalysis, thiapyrylium salts, indoles, 1,3cyclohexadienes

\section{Introduction}

The Diels-Alder reaction is a well established synthetic method in organic chemistry, which allows creation of two new carbon-carbon bonds, leading to the formation of six-membered rings. However, this reaction is generally inefficient when both diene (D) and dienophile (A) are electron-rich compounds. ${ }^{1,2}$ Several strategies have been devised to overcome this limitation. ${ }^{3-7}$ One of them makes use of triarylpyrylium salts as electron transfer photocatalysts. ${ }^{6}$ Indole (5) 
quenches the fluorescence of triarylpyrylium salts; therefore, it has been suggested that the photocatalysts singlet excited state is involved in the cycloaddition process.

Electrochemical and photophysical properties, such as reduction potential, fluorescence quantum yield and T-T absorption spectrum of pyrylium salts, are often influenced by the substitution pattern of the aryl groups and by the nature of the heteroatom in the heterocycle. ${ }^{8}$ The value of the oxidation potential seems to be a major factor conditioning the product yields and product distribution. ${ }^{8}$ In particular, it has been published ${ }^{6}$ that the Diels-Alder reaction between phellandrene (4b) and $\mathbf{5}$ improves from $57 \%$ to $67 \%$ when tris(4methoxyphenyl)pyrylium $\mathbf{3}\left(E_{\text {red }}=-0.52 \mathrm{~V}\right.$ vs SCE$)$ is used instead of $\mathbf{2}\left(E_{\text {red }}=-0.29 \mathrm{~V} \text { vs SCE }\right)^{9}$ as catalyst (Chart 1, Scheme 1). Also, the fluorescence quantum yield of $3\left(\Phi_{f}=0.97\right)$, the highest among the studied pyrylium salts, could contribute to the increased reaction efficiency. Concerning the effect of spin multiplicity of the photocatalyst on this cycloaddition, no data has been reported as yet. Taking into account that the sulfur atom in thiapyrylium salt $\mathbf{1}$ exerts an internal heavy atom effect, leading to a very high intersystem crossing quantum yield ( $\Phi_{i s c}=$ 0.97), ${ }^{10}$ it appeared of interest to study the role of this factor in the cycloaddition between InHs and 1,3-cyclohexadienes (CHDs). Herein, we report that, as a matter of fact, 1 photocatalyzes this Diels-Alder reaction with similar efficiency as the oxa-analogues, though with a lower stereoselectivity. Steady-state and time resolved studies agree well with the involvement of both the singlet and the triplet excited states.

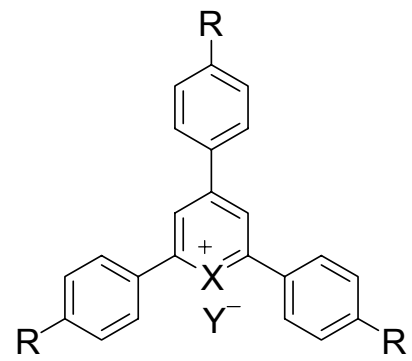

$1 \mathrm{R}=\mathrm{H}, \mathrm{X}=\mathrm{S}, \mathrm{Y}=\mathrm{ClO}_{4}$

$2 \mathrm{R}=\mathrm{H}, \mathrm{X}=\mathrm{O}, \mathrm{Y}=\mathrm{BF}_{4}$

$3 \mathrm{R}=\mathrm{OCH}_{3}, \mathrm{X}=\mathrm{O}, \mathrm{Y}=\mathrm{BF}_{4}$

Chart 1. Structure of the photocatalysts. 


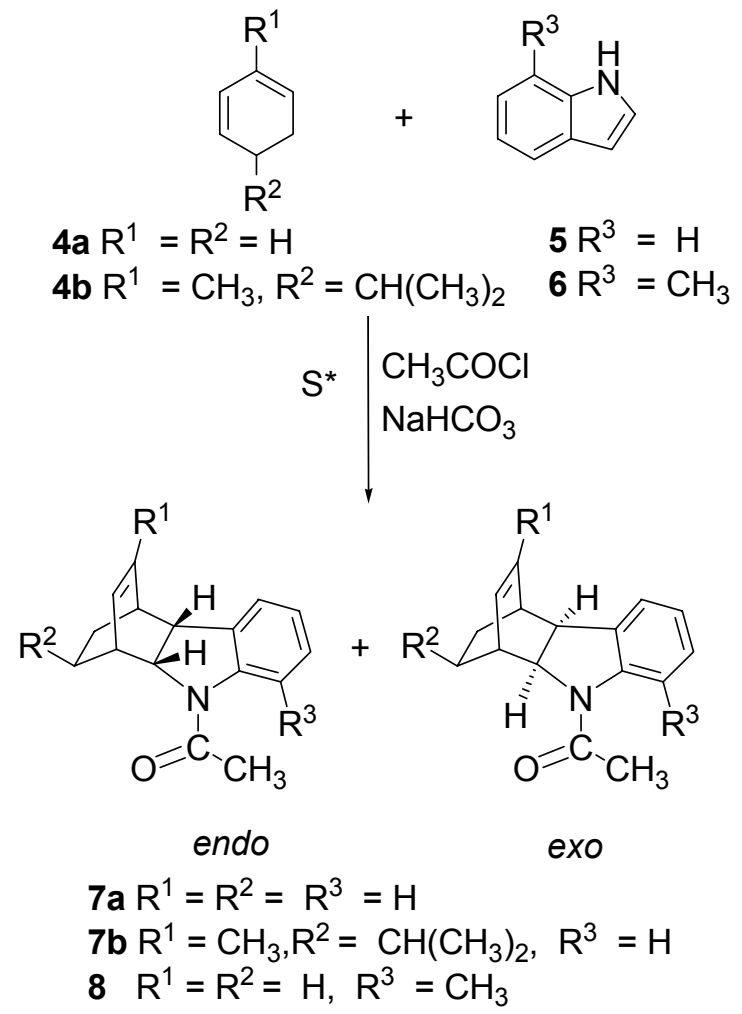

Scheme 1. Diels-Alder reaction photocatalyzed by pyrylium salts.

\section{Results}

\section{Steady-state irradiation}

The UV-visible absorption spectra of $\mathbf{1}$ in the absence and in the presence of indole (5) and CHD (4a) were obtained in dichloromethane. The absence of any characteristic charge transfer (CT) absorption in the presence of the quenchers agreed with the absence of ground-state complexation (Figure 1). ${ }^{10}$ The intense absorption band at $\lambda>320 \mathrm{~nm}$ allows selective excitation of the thiapyrylium salt in the presence of InHs and CHDs. 


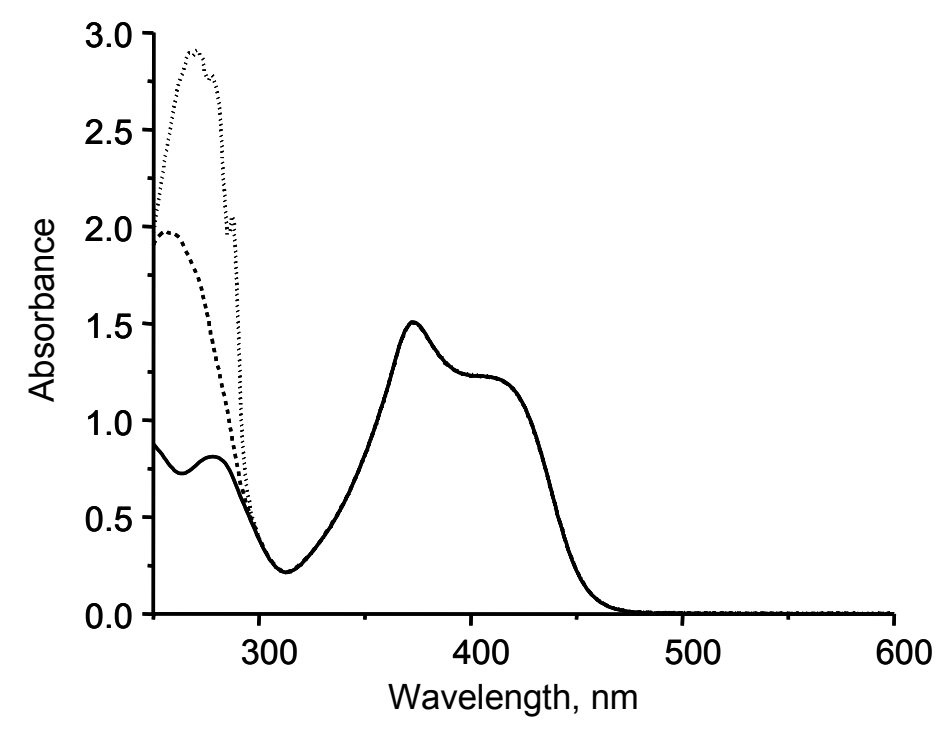

Figure 1. Absorption spectra of $1\left(1.6 \times 10^{-5} \mathrm{M}\right)$ in the absence $(-)$ and in the presence of $5(\cdots)$ $\left(3,2 \times 10^{-4} \mathrm{M}\right)$ or $\mathbf{4 a}(---)\left(6,4 \times 10^{-4} \mathrm{M}\right)$ in dichloromethane.

Product studies were performed by irradiation (UVA lamp emitting at $400 \mathrm{~nm} \geq \lambda \geq 320$ $\mathrm{nm})$ of deaerated dichloromethane solutions of $\mathbf{1}(0.001 \mathrm{M}), \mathbf{4 a}(0.04 \mathrm{M})$ and $\mathbf{5}(0.02 \mathrm{M})$ for $6 \mathrm{~h}$. Previous reports on this reaction using triarylpyrylium salts as photocatalysts have stressed the need to protect the amino group of the nascent photoadduct. ${ }^{6}$ Therefore, an acylation reagent (acetyl chloride $/ \mathrm{NaHCO}_{3}$ ) was also added to the reaction mixture prior to irradiation.

Indeed, the acylated Diels-Alder products $7 \mathbf{a}$ were obtained in ca. $62 \%$ yield, with an endo:exo ratio close to $1.8: 1$ (Scheme 1 and run 1 of Table 1). Slightly higher yield (70\%) and selectivity (endo:exo 3.3/1.0) has been achieved using 2 as photocatalyst (run 2, Table 1). ${ }^{6}$ As in the case of using pyrylium salts $\mathbf{2}$ or $\mathbf{3}$ as photosensitizers, no [2+2] cross-cycloaddition products were detected. ${ }^{6}$

Electron transfer from $4 \mathbf{a}$ to $\mathbf{1}$ led to CHD dimers as by-products ( $7 \%$ relative to the initial amount of 1,3-cyclohexadiene), with an isomer distribution typical of radical cation dimerizations. The major product was the endo-[4+2] dimer, which agrees with the high selectivity found when 1 was used for the dimerization of CHD in dichloromethane. ${ }^{8}$

Control experiments showed that [4+2] cross-cycloaddition did not occur in the absence of $\mathbf{1}$. Also, irradiation of $\mathrm{N}$-acetylindole and cyclohexadiene in the presence of $\mathbf{1}$ gave only traces amounts of $7 \mathbf{a}$.

$\alpha$-Phellandrene (racemic mixture) 4b was also tested in the cross-cycloaddition with $\mathbf{5}$ photoinduced by $\mathbf{1}$. This 2,5-disubstituted CHD derivative led to a similar yield of Diels-Alder products as $\mathbf{4 a}$ (compare runs 1 and 3 of Table 1). However, 2 was less efficient with $\mathbf{4 b}$ than $\mathbf{4 a}$, and formation of $\mathbf{7 b}$ occurred with a $57 \%$ yield. ${ }^{6}$ Better efficiency and selectivity were achieved with the stronger electron acceptor pyrylium salt $3^{6}$ (run 5 of Table 1), which also has a higher 
fluorescence quantum yield ( $\Phi_{f}=0.97$ for 3 vs 0.47 for 2 ). The relative stereoselectivity found in the pyrylium salts-promoted cycloaddition follows the order $\mathbf{1}<\mathbf{2}<\mathbf{3}$.

Table 1. Diels-Alder reaction between indoles and cyclohexadienes photocatalyzed by pyrylium salts in dichloromethane.

\begin{tabular}{cccccc}
\hline Run & Cat. & Diene & Indole & $\begin{array}{c}\text { Diels-Alder } \\
\text { Yield (\%) }\end{array}$ & endo:exo $^{\mathrm{b}}$ \\
\hline 1 & $\mathbf{1}$ & $\mathbf{4 a}$ & $\mathbf{5}$ & $\mathbf{7 a ( 6 2 )}$ & $1.8: 1.0$ \\
2 & $\mathbf{2}$ & $\mathbf{4 a}$ & $\mathbf{5}$ & $\mathbf{7 a}^{\mathrm{a}(70)^{\mathrm{c}}}$ & $3.3: 1.0$ \\
3 & $\mathbf{1}$ & $\mathbf{4 b}$ & $\mathbf{5}$ & $\mathbf{7 b}^{\mathrm{b}}(66)$ & $1.7: 1.0$ \\
4 & $\mathbf{2}$ & $\mathbf{4 b}$ & $\mathbf{5}$ & $\mathbf{7 b}(57)^{\mathrm{c}}$ & $2.4: 1.0$ \\
5 & $\mathbf{3}$ & $\mathbf{4 b}$ & $\mathbf{5}$ & $\mathbf{7 b}(67)^{\mathrm{c}}$ & $4.6: 1.0$ \\
6 & $\mathbf{1}$ & $\mathbf{4 a}$ & $\mathbf{6}$ & $\mathbf{8}(33)$ & $1.9: 1.0$ \\
\hline
\end{tabular}

${ }^{a}$ Spectroscopically determined based on the initial amount of indole, no other products derived from indole were detected. If calculations are done based on initial CHD, the yields are halved. ${ }^{\mathrm{b}}$ Determined by comparing the integrals of the characteristic ${ }^{1} \mathrm{H}-\mathrm{NMR}$ signals between 4.2 and $4.8 \mathrm{ppm}$ for the two rotamers of each stereoisomer. ${ }^{\mathrm{c}}$ reference 6.

On the other hand, experiments previously performed with $\mathbf{2}$ have shown that 2-methylindole does not lead to photoadducts with CHD in the pyrylium salt-photocatalyzed process, while methyl 3-indolylacetate reacts only sluggishly. ${ }^{6}$ These results have been attributed to steric hindrance at the attacked indole positions. In order to check the possible influence of steric effects at other positions, 7-methylindole (6) was used as dienophile with $\mathbf{1}$ as photocatalyst; this provided $\mathbf{8}^{11}$ in a moderate yield and similar stereoselectivity as $7 \mathbf{a}$ (run 6 , Table 1).

\section{Fluorescence studies}

To get some insight into the photophysical behavior of the excited singlet state of 1, its quenching by 5 and $\mathbf{4 a}$ was examined in dichloromethane. Indeed, the fluorescence $\left(\lambda_{\max }=464\right.$ $\mathrm{nm}$ in dichloromethane, Figure 2) of $\mathbf{1}$ was efficiently quenched by both types of substrates. The fluorescence quenching rate constants by indole $\left({ }^{1} k_{q}=2.2 \times 10^{10} \mathrm{M}^{-1} \mathrm{~s}^{-1}\right)$ and cyclohexadiene $\left({ }^{1} k_{q}\right.$ ' $=0.7 \times 10^{10} \mathrm{M}^{-1} \mathrm{~s}^{-1}$ ) were obtained from the Stern-Volmer analysis (eqs 1-4, Figure 3), using the singlet lifetime of $1 \tau_{\mathrm{f}}=3.6 \mathrm{~ns}^{8}{ }^{8}$ 


$$
\begin{aligned}
& I_{0} / I_{1}=1+K_{S V}[\operatorname{lnH}](1) \\
& K_{S V}=\tau_{f}^{1} k_{\mathrm{q}} \\
& I_{0} / I_{2}=1+K_{S V}[\mathrm{CHD}](3) \\
& K_{S V^{\prime}}=\tau_{f}^{1} K_{\mathrm{q}}^{\prime}
\end{aligned}
$$

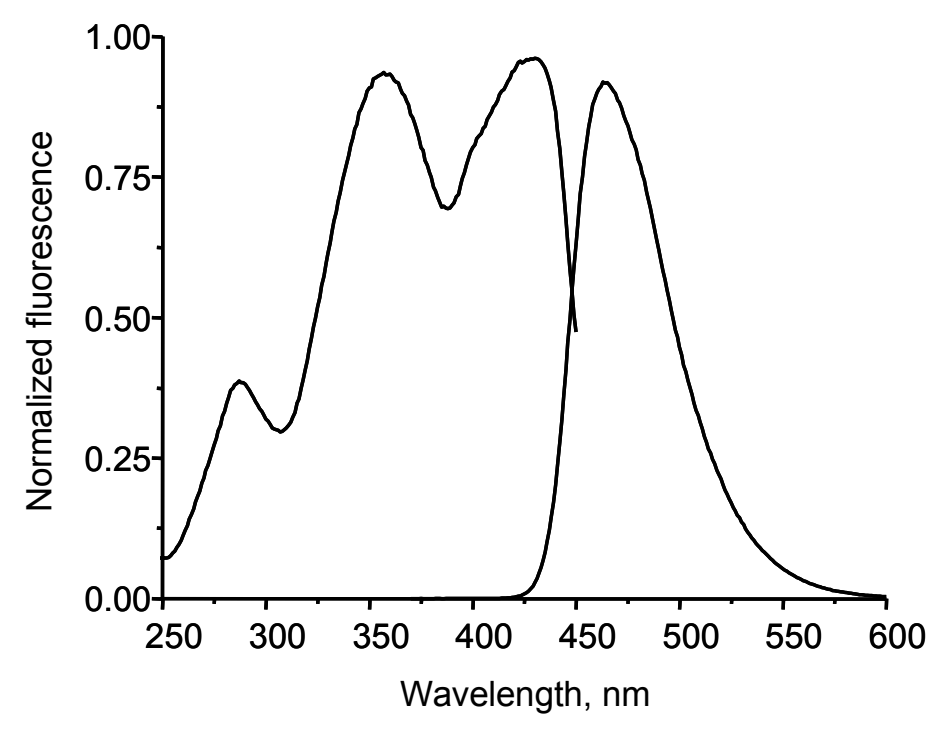

Figure 2. Normalized fluorescence emission (right) and excitation spectra (left) of $1\left(4,7 \times 10^{-5}\right.$ M) in dichloromethane.

The free energy change associated with electron transfer from indole to the excited singlet state of the thiapyrylium salt was estimated according to the Rehm-Weller equation (eq 5)

$$
\Delta G_{\mathrm{ET}}^{0}=E_{\mathrm{ox}}\left(\mathrm{D} / \mathrm{D}^{+}\right)-E_{\mathrm{red}}\left(\mathrm{A}^{-} / \mathrm{A}\right)-E_{0,0} *+2.6 \mathrm{eV} / \varepsilon-0.13 \mathrm{eV}
$$

where $E_{0,0} *$ is the singlet energy of the thiapyrylium salt $\left(66.0 \mathrm{kcal}^{\mathrm{mol}} \mathrm{m}^{-1}\right){ }^{12}$

Thus, the free energy change, $\Delta G_{\mathrm{ET}}^{0}$, for the electron transfer process in dichloromethane was estimated as $-32.1 \mathrm{kcal}^{\mathrm{mol}}{ }^{-1}$ using the known half-wave oxidation potential of indole (around $1.1 \mathrm{~V} v s \mathrm{SCE})^{13}$ and half-wave reduction potential of $\mathbf{1}\left(-2.53 \mathrm{~V} v_{S} \mathrm{SCE}\right) .^{14}$

The rate constants for quenching of the fluorescence of pyrylium salt $\mathbf{2}$ by indole and cyclohexadiene were also determined $\left(5.2 \times 10^{10} \mathrm{M}^{-1} \mathrm{~s}^{-1}\right.$ and $1.0 \times 10^{10} \mathrm{M}^{-1} \mathrm{~s}^{-1}$, respectively). In addition, the free energy change associated with electron transfer from 5 to the excited singlet state of the pyrylium salt was estimated at $-30.2 \mathrm{kcal}_{\mathrm{mol}} \mathrm{l}^{-1}$.

\section{Intersystem crossing contribution to deactivation of the thiapyrylium singlet}

To quantify the amount of triplet excited state of $\mathbf{1}$ formed, the relative intersystem crossing yields under the employed reaction conditions were estimated taking into account the main 
deactivation pathways of the singlet excited state: fluorescence emission, fluorescence quenching, and intersystem crossing. Competition between them should be governed by the respective rate constants and by the quencher concentration.

The rate constant of fluorescence emission $\left(k_{f}\right)$ and intersystem crossing $\left(k_{I S C}\right)$ are intrinsic properties of the photocatalyst. The values for 1 were calculated from eqs 6 and $7\left(\tau_{\mathrm{f}}=3.6 \mathrm{~ns}^{8}\right.$ and $\Phi_{\mathrm{f}}=0.03^{15}$ ) and found to be $k_{f}=0.8 \times 10^{7} \mathrm{~s}^{-1}$ and $k_{I S C}=2.7 \times 10^{8} \mathrm{~s}^{-1}$.

$$
\begin{aligned}
& \tau_{f} k_{\mathrm{f}}=\Phi_{\mathrm{f}}(6) \\
& \tau_{f} k_{\mathrm{ISC}}=\Phi_{\mathrm{ISC}}(7)
\end{aligned}
$$
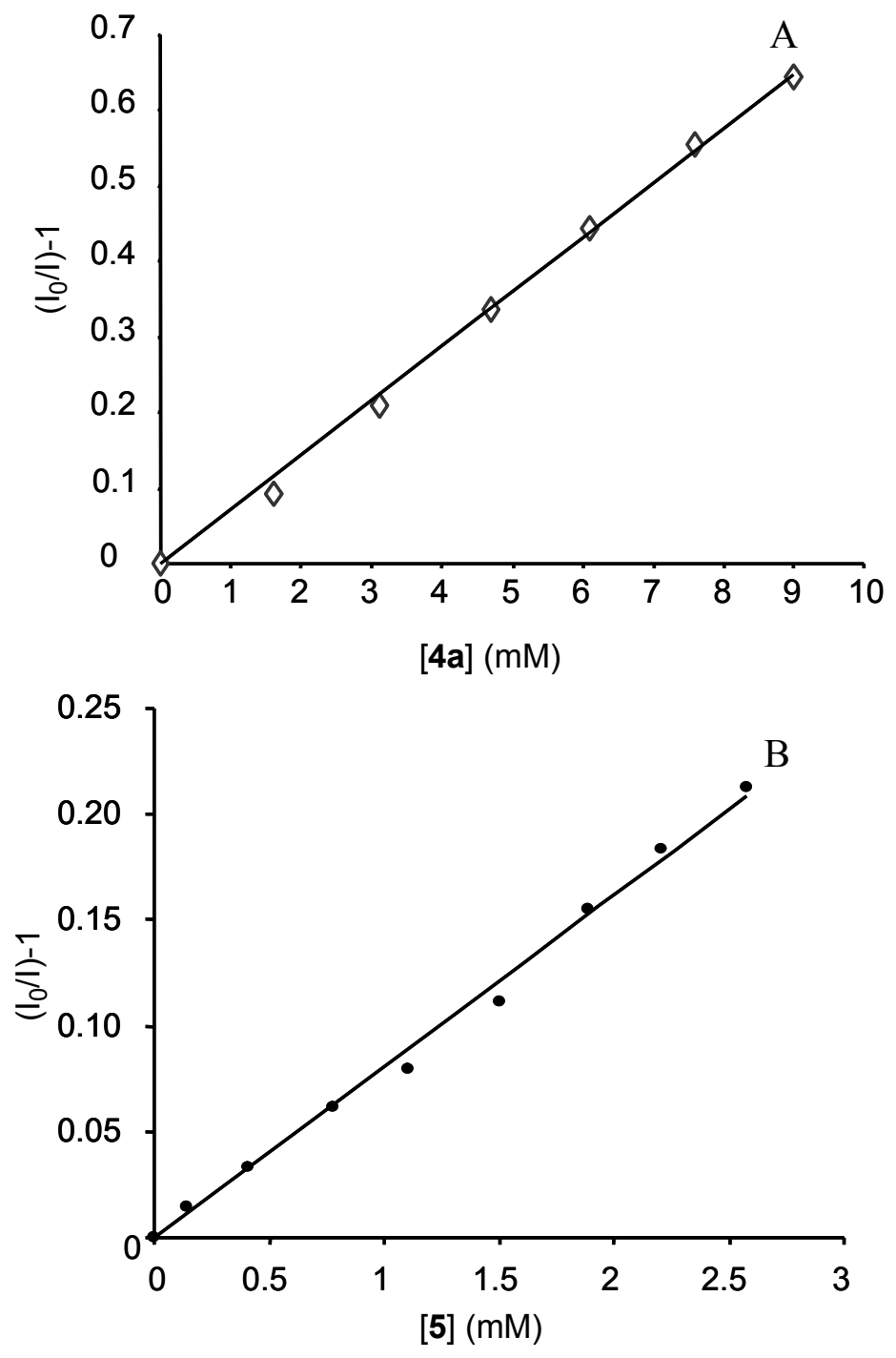

Figure 3. Stern-Volmer plots for fluorescence quenching of $\mathbf{1}\left(4.7 \times 10^{-5} \mathrm{M}\right)$ by A: $\mathbf{4 a}$ and B: $\mathbf{5}$ in dichloromethane. 
From these data and the rate constants for quenching of the thiapyrylium salt fluorescence, the contributions of the different singlet deactivation pathways can be estimated (eqs 8-12).

$$
\begin{aligned}
& k_{s}=k_{\mathrm{f}}+k_{\mathrm{ISC}}+{ }^{1} k_{\mathrm{q}}[\mathrm{InH}]+{ }^{1} k_{\mathrm{q}}{ }^{\prime}[\mathrm{CHD}](8) \\
& \text { Residual Fluorescence: } k_{f} / k_{\mathrm{s}}=0.8 \% \text { (9) } \\
& \text { Quenching of fluorescence by } 5:{ }^{1} k_{q}[\mathrm{InH}] / k_{\mathrm{s}}=44.0 \% \quad(10) \\
& \text { Quenching of fluorescence by } 4 \mathrm{a}:{ }^{1} k_{q}[\mathrm{CHD}] / k_{\mathrm{s}}=28.0 \% \\
& \text { Intersystem crossing: } k_{I S C} / k_{\mathrm{s}}=27.0 \%
\end{aligned}
$$

Thus, it is clear that i) intersystem crossing to the triplet excited state has a considerable contribution to singlet deactivation, and ii) the triplet excited state of the photocatalyst is sufficiently populated. It may behave as electron acceptor towards indole, since this process is thermodynamically feasible. Accordingly, the free energy change for the electron transfer process in dichloromethane was estimated as $\Delta G_{\mathrm{ET}}^{0}=-18.1 \mathrm{kcal} . \mathrm{mol}^{-1}$ using eq. 5 , where $E_{0,0} *$ is the triplet energy of the thiapyrylium salt $\left(52.0 \mathrm{kcal}^{\mathrm{m}} \mathrm{mol}^{-1}\right){ }^{14}$

Similar calculations with 2 led to a relative intersystem crossing yield of $8 \%$.

\section{Quenching of the photocatalyst triplet}

Laser excitation (Nd:YAG, $10 \mathrm{~ns}$ laser pulse, $\lambda_{\text {exc }}=355 \mathrm{~nm}$ ) of dichloromethane solutions containing thiapyrylium salt $1\left(1.6 \times 10^{-5} \mathrm{M}\right)$ gave rise to a depletion of the ground state absorption between 390 and $440 \mathrm{~nm}$. This was followed by the appearance of a broad transient absorption between 460 and $600 \mathrm{~nm}$, with a lifetime of ca. $6.9 \mu$ s (Figure 4), which was assigned to the known T-T transition of $\mathbf{1}^{15}$

Both diene 4a and dienophile 5 quenched the triplet excited state of the thiapyrylium salt; the rate constants for triplet quenching $\left({ }^{3} k_{q}\right.$ and $\left.{ }^{3} k_{q}\right)$ were measured by monitoring the decay of the $\mathrm{T}$-T transition at $500 \mathrm{~nm}$, using increasing amounts of the quencher and recording the SternVolmer plots (Figure 5). Thus, values of $8.8 \times 10^{9} \mathrm{M}^{-1} \mathrm{~s}^{-1}$ and $4.7 \times 10^{8} \mathrm{M}^{-1} \mathrm{~s}^{-1}$ were calculated for 5 and $\mathbf{4 a}$, respectively. 


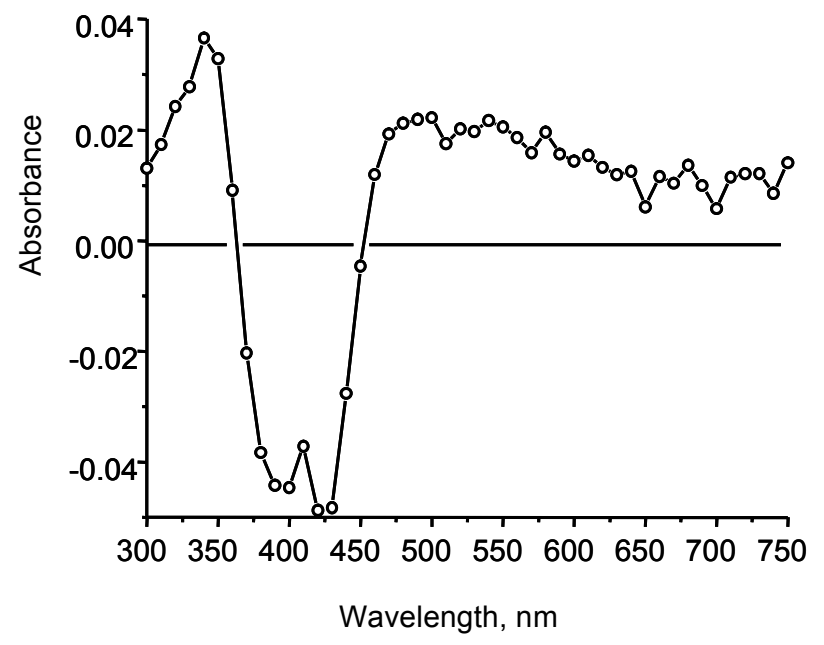

Figure 4. Transient absorption spectra recorded following laser excitation ( $355 \mathrm{~nm})$ of $\mathbf{1}$ (1.6 x $10^{-5} \mathrm{M}$ ) in deaerated dichloromethane $3 \mu$ s after the laser pulse.
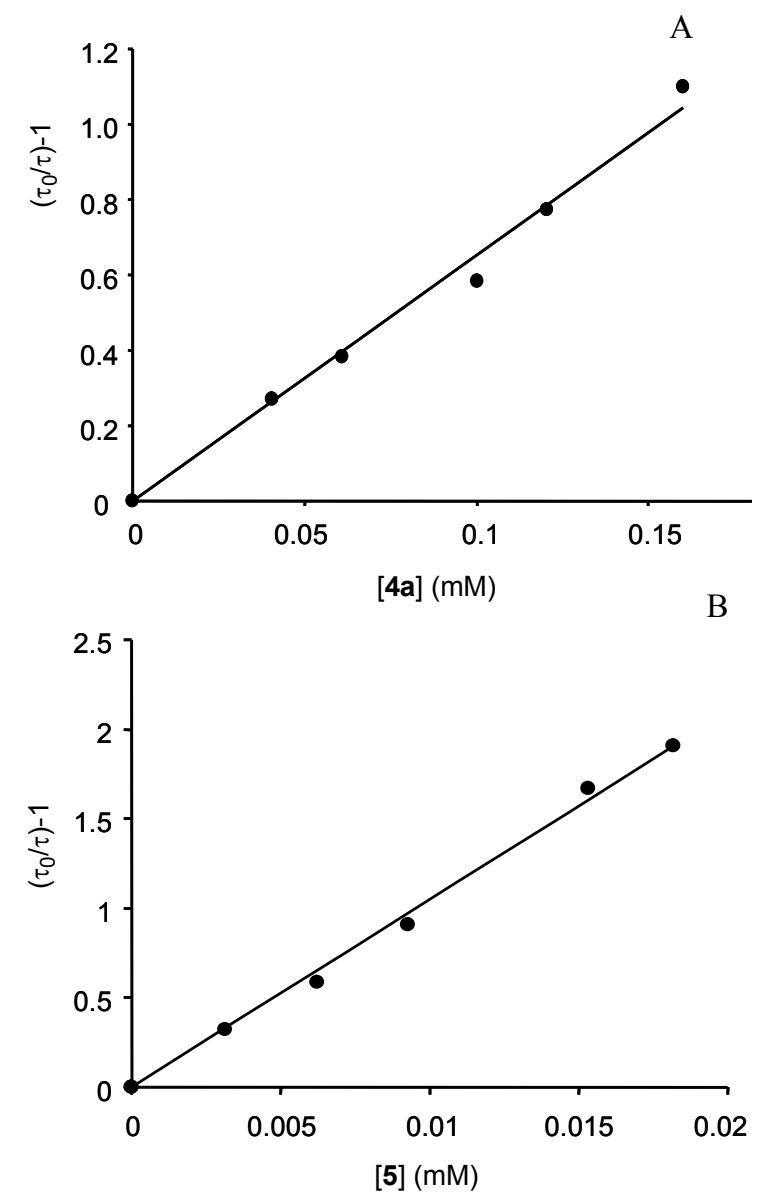

Figure 5. Stern-Volmer plots for triplet quenching of $\mathbf{1}\left(1.6 \times 10^{-5} \mathrm{M}\right)$ by A: $4 \mathbf{a}$ and B: 5 in dichloromethane. 


\section{Discussion}

Photoinduced electron transfer (PET) processes involve exchange of one electron between the excited state of the photocatalyst (singlet or triplet) and the quencher, generating a radical ion pair. The efficiency of the subsequent processes can depend on spin multiplicity. ${ }^{16}$ Triplets are usually longer-lived, which allows the partners to diffuse and react. Moreover, back electron transfer is slow within the triplet radical ion pairs, facilitating cage escape. Pyrylium salts are well-established PET photocatalysts; depending on the substitution pattern they can generate singlet or triplet radical ions pairs. The use of thiapyrylium salt $\mathbf{1}$, with the highest intersystem crossing quantum yield, allows determining the effect of the triplet on the Diels-Alder reaction of InHs with CHDs.

The fluorescence quenching of $\mathbf{1}$ by indole and the large free energy changes associated with electron transfer from indole to the excited singlet state of the thiapyrylium salt strongly suggest that this process takes place quite efficiently from $\mathbf{5}$ to the singlet excited state of $\mathbf{1}$. Nevertheless, the triplet is also populated and efficiently quenched by $\mathbf{5}$, being electron transfer thermodynamically feasible. Therefore, the electron transfer from indole not only to the singlet excited state of $\mathbf{1}$ but also to the triplet can be an important primary process.

The fact that the yields of the Diels-Alder reaction are similar for $\mathbf{1}$ and 2, but with reduced stereoselectivity when using the former, could agree with the much higher contribution of the photocatalyst triplet excited state in the case of the thiapyrylium salt.

\section{Conclusions}

The [4+2]-cycloaddition of indoles with 1,3-cyclohexadienes can be efficiently photocatalyzed by thiapyrylium salts. The experimental results agree with partial involvement of the triplet excited state in the electron transfer process. Cross-cycloadducts yields are similar to those obtained using the oxa-analogues, though the process is less stereoselective. So far, this is the only reported example of thiapyrylium photocatalyzed cycloaddition of indoles with cyclohexadienes.

\section{Experimental Section}

General Procedures. ${ }^{1} \mathrm{H}$ and ${ }^{13} \mathrm{C}$ NMR spectra were recorded in a $(400 \mathrm{MHz})$ Brucker spectrometer; chemical shifts $(\delta)$ are reported in ppm relative to TMS. The coupling constants $(J)$ are in hertz $(\mathrm{Hz})$. Dichloromethane was dried over $\mathrm{CaCl}_{2}$ and distilled over $\mathrm{CaH}_{2}$ prior use. All reagents were commercially available and used without further purification, except 1,3cyclohexadiene (distilled over $\mathrm{NaBH}_{4}$ ). Column chromatography was performed on silica gel 60 
(0.040-0.063 mm). GC/MS data are taken under electron ionization (EI) conditions of about 30 eV. High-resolution mass spectra (HRMS) were conducted at the SCSIE in Valencia, Spain.

Steady-state spectroscopic measurements. UV/vis absorption measurements were performed on a Agilent 8453 spectrometer. Fluorescence emission and excitation spectra were recorded on a Photon Technology International (PTI) LPS-220B fluorimeter. Measurements were done at 25 ${ }^{\circ} \mathrm{C}$ in cuvettes of $1 \mathrm{~cm}$ path length.

Laser flash photolysis. Laser flash photolysis experiments were carried out by using the third harmonics $(355 \mathrm{~nm})$ of a pulsed Nd:YAG laser. The pulse duration was $10 \mathrm{~ns}$, and the energy of the laser beam was $18 \mathrm{~mJ} /$ pulse. A xenon lamp was employed as detecting light source. The laser apparatus consisted of the pulsed laser, the Xe lamp, a monochromator, a photomultiplier (PMT) system and an oscilloscope. The output signal was transferred to a personal computer for data analysis.

Standard procedure for the Diels-Alder reactions. To a deaerated dichloromethane $(5 \mathrm{~mL})$ solution of indole $(0.02 \mathrm{M}), 1,3$-cyclohexadiene $(0.04 \mathrm{M})$ and powdered $\mathrm{NaHCO}_{3}(18 \mathrm{mg})$, acetyl chloride $(0.02 \mathrm{M})$ was added. Then, the thiopyrylium salt $(0.001 \mathrm{M})$ was added under a nitrogen atmosphere. Irradiation was carried out in a photoreactor equipped with eigth UVA lamps (400 nm $\geq \lambda \geq 320 \mathrm{~nm}, \lambda_{\max }$ centered at $350 \mathrm{~nm}$ ). The solutions were irradiated for $6 \mathrm{~h}$, then filtered, washed with water and dried over $\mathrm{Na}_{2} \mathrm{SO}_{4}$. The solvent was evaporated and the crude was analyzed by ${ }^{1} \mathrm{H}-\mathrm{NMR}$.

\section{Acknowledgements}

We thank the Ministerio de Educación y Ciencia (Project CTQ2005-00569, doctoral fellowship to M.G.B.) and Generalitat Valenciana (GV-ACOMP06/134) for generous support of this work.

\section{References and Footnotes}

1. (a) An interesting exception is the Diels-Alder reaction between $\mathrm{C}_{60}$ and Danishefsky's dienes: Mikami, K.; Matsumoto, S.; Okubo, Y.; Fujitsuka, M.; Ito, O.; Suenobu, T.; Fukuzumi, S. J. Am. Chem. Soc. 2000, 122, 2236. (b) Strained dienophiles can also lead to [4+2] adducts: Collins, S. K.; Yap, G. P. A.; Fallis, A. G. Org. Lett. 2002, 4, 11.

2. Some thermal Diels-Alder reactions between electron-rich components are metal-catalyzed processes: Hilt, G.; Smolko, K. I. Synthesis 2002, 686.

3. (a) Mattay, J.; Trampe, G.; Runsink, J. Chem. Ber. 1988, 121, 1991. (b) Mattay, J.; Vondenhof, M.; Denig, R. Chem. Ber. 1989, 122, 951. 
4. (a) Pabon, R. A.; Bellvile, D. J.; Bauld, N. L. J. Am. Chem. Soc. 1983, 105, 5158. (b) Schmittel, M.; von Seggern, H. J. Am. Chem. Soc. 1993, 115, 2165. (c) Bauld, N. L.; Yang, J.; Gao, D. J. Chem.Soc., Perkin Trans. 2 2000, 207.

5. (a) Calhoun, G. C.; Schuster, G. B. Tetrahedron Lett. 1986, 27, 911. (b) Akbulut, N.; Hartsough, D.; Kim, J. I.; Schuster, G. B. J. Org. Chem. 1989, 54, 2549. (c) Kim, J. I.; Schuster, G. B. J. Am. Chem. Soc. 1992, 114, 9309.

6. (a) Gieseler, A.; Steckhan, E.; Wiest, O.; Knoch, F. J. Org. Chem. 1991, 56, 1405. (b) Wiest, O.; Steckhan, E. Tetrahedron Lett. 1993, 34, 6391.

7. (a) Pérez-Prieto, J.; Stiriba, S.-E.; González-Béjar, M.; Domingo, L. R.; Miranda, M. A. Org. Lett. 2004, 6, 3905. (b) González-Béjar, M.; Stiriba, S.-E.; Domingo, L. R.; PérezPrieto, J.; Miranda, M. A. J. Org. Chem. 2006, 71, 6932.

8. Martiny, M.; Steckhan, E.; Esch, T. Chem. Ber. 1993, 126, 1671.

9. Saeva, F. D.; Olin, G. R. J. Am. Chem. Soc. 1980, 102, 299.

10. Jayanthi, S. S.; Ramamurthy, P. J. Phys. Chem. A 1998, 102, 511.

11. Characterized by comparison with analogous cycloadducts. Endo-8: ${ }^{1} \mathrm{H}$ NMR (400 MHz, $\left.\mathrm{CDCl}_{3}\right): \delta 0.9-1.8(\mathrm{~m}, 4 \mathrm{H}), 2.2(\mathrm{~s}, 3 \mathrm{H}), 2.3(\mathrm{~s}, 3 \mathrm{H}), 2.9(\mathrm{~m}, 1 \mathrm{H}), 3.0(\mathrm{~m}, 1 \mathrm{H}), 3.8(\mathrm{dd}, J(\mathrm{H}$, $\mathrm{H})=8.8,3.2 \mathrm{~Hz}, 1 \mathrm{H}), 4.4(\mathrm{~d}, J(\mathrm{H}, \mathrm{H})=7.6 \mathrm{~Hz}, 1 \mathrm{H}), 5.7(\mathrm{t}, J(\mathrm{H}, \mathrm{H})=7.4 \mathrm{~Hz}, 2 \mathrm{H}), 6.0(\mathrm{t}, J$ $(\mathrm{H}, \mathrm{H})=7.4 \mathrm{~Hz}, 2 \mathrm{H}), 6.9-7.0(\mathrm{~m}, 3 \mathrm{H})$; $\mathrm{MS}(30 \mathrm{eV}): m / z(\%): 253$ (12) $\left[\mathrm{M}^{+}\right], 173$ (44), 131 (100); FAB-MS calcd for $\mathrm{C}_{17} \mathrm{H}_{20} \mathrm{NO}$ : $\left[\mathrm{M}^{+}+1\right]$, 254.1545. Found: 254.1544. Exo-8: relevant ${ }^{1} \mathrm{H}$ NMR $\left(400 \mathrm{MHz}, \mathrm{CDCl}_{3}\right)$ signals: $\delta 2.8(\mathrm{~m}, 1 \mathrm{H}), 2.9(\mathrm{~m}, 1 \mathrm{H}), 3.6(\mathrm{~m}, 1 \mathrm{H}), 4.3(\mathrm{~d}, J(\mathrm{H}$, $\mathrm{H})=7.6 \mathrm{~Hz}, 1 \mathrm{H})$; MS (30 eV): $m / z(\%): 253(9)\left[\mathrm{M}^{+}\right], 173$ (53), 131 (100).

12. Miranda, M. A.; Izquierdo, M. A.; Pérez-Ruiz, R. J. Phys. Chem. A 2003, 107, 2478.

13. Pérez-Prieto, J.; Bosca, F.; Galian, R. E.; Lahoz, A.; Domingo, L. R.; Miranda, M. A. J. Org. Chem. 2003, 68, 5104.

14. Miranda, M. A.; García, H. Chem. Rev. 1994, 94, 1063.

15. Akaba, R.; Kamata, M.; Koike, A.; Mogi, K-I.; Kuriyama, Y.; Sakuragi, H. J. Phys. Org. Chem. A 1997, 10, 861.

16. Julliard, M.; Chanon, M. Chem. Rev. 1983, 83, 425. 